\title{
Observing recommendations for JWST MIRI users
}

\section{Macarena García Marín, George Rieke, Michael Ressler, Dan Dicken, Tom Greene, et al.}

Macarena García Marín, George Rieke, Michael Ressler, Dan Dicken, Tom Greene, Jane Morrison, Sarah Kendrew, Stacey Alberts, Michael Regan, David Law, Ori Fox, Tea Temim, Dean Hines, Pierre-Olivier Lagage, Pamela Klaassen, John Pye, Gillian Wright, Alistair Glasse, "Observing recommendations for JWST MIRI users," Proc. SPIE 10704, Observatory Operations: Strategies, Processes, and Systems VII, 107041I (10 July 2018); doi: $10.1117 / 12.2314319$

Event: SPIE Astronomical Telescopes + Instrumentation, 2018, Austin, Texas, United States 


\title{
Observing recommendations for JWST MIRI users
}

\author{
Macarena García Marín ${ }^{\mathrm{a}}$, George Rieke ${ }^{\mathrm{b}}$, Michael Ressler ${ }^{\mathrm{c}}$, Dan Dicken ${ }^{\mathrm{d}}$, Tom Greene ${ }^{\mathrm{e}}$, Jane \\ Morrison $^{\mathrm{b}}$, Sarah Kendrew ${ }^{\mathrm{a}}$, Stacey Alberts ${ }^{\mathrm{b}}$, Michael Regan ${ }^{\mathrm{f}}$, David Law ${ }^{\mathrm{f}}$, Ori Fox ${ }^{\mathrm{f}}$, Tea \\ Temim $^{\mathrm{f}}$, Dean Hines ${ }^{\mathrm{f}}$, Pierre-Olivier Lagage ${ }^{\mathrm{d}}$, Pamela Klaassen ${ }^{\mathrm{g}}$, John Pye ${ }^{\mathrm{h}}$, Gillian Wright ${ }^{\mathrm{g}}$, \\ and Alistair Glasse ${ }^{\mathrm{g}}$ \\ ${ }^{a}$ European Space Agency, 3700 San Martin Drive, Baltimore, USA \\ bSteward Observatory 272, University of Arizona, Tucson, USA \\ ${ }^{\mathrm{c}}$ Jet Propulsion Laboratory, 4800 Oak Grove Drive, Pasadena, USA \\ dDépartement d'Astrophysique, CEA, Saclay, France \\ eNASAs Ames Research Center, MS 2456, Moffett Field, USA \\ ${ }^{\mathrm{f}}$ Space Telescope and Science Institute, 3700 San Martin Drive, Baltimore, USA \\ 'UK Astronomy Technology Centre, Royal Observatory, Edinburgh, UK \\ ${ }^{\mathrm{h}}$ University of Leicester, Dept of Physics \& Astronomy, Leicester, UK
}

\begin{abstract}
The Mid-Infrared Instrument (MIRI), a result of the collaborative work of a consortium of European and US institutes, is the only Mid-IR science instrument on the James Webb Space Telescope (JWST). The combination of MIRI's sensitivity and angular resolution over the 5-28.5 $\mu \mathrm{m}$ wavelength range will enable investigations into many different science topics, ranging from the local to the high-redshift Universe. The MIRI team has defined and published a set of "Recommended Strategies" to help observers optimally plan and execute their science programs. Some of these recommendations are generic and applicable to any science case; others are tailored to specific observing modes. Here we summarize key generic recommendations for MIRI observers, with emphasis on detector usage. All this information is available to observers as part of the James Webb Telescope User's Documentation System and will be updated as needed. ${ }^{1}$
\end{abstract}

Keywords: JWST, MIRI, Observations planning

\section{INTRODUCTION}

The MIRI Optics Module (OM) combines an innovative instrument modular design ${ }^{2}$ with three arsenic-doped silicon impurity band conduction detectors $\left(\mathrm{see}^{3,4}\right)$. The instrument will provide imaging, ${ }^{5}$ coronagraphy ${ }^{6}$ lowresolution slit and slitless (optimized for Time Series Observations) spectroscopy (Low Resolution Spectrometer, LRS), ${ }^{7}$ and medium resolution integral-field spectroscopy (Medium Resolution Spectrometer, MRS) ${ }^{8}$ at subarcsecond angular resolution and unprecedented sensitivity in the MIR regime. ${ }^{9}$ The imaging module (imaging, coronagraphy and LRS) makes use of one detector, the MRS uses two. This wide suite of observing modes will enable a wealth of scientific investigations. ${ }^{10}$ The MIRI detectors have heritage to those used on Spitzer, but the multiple MIRI observational modes necessitate a significant number of decisions to optimize the science return. To prepare the observations, users will have to make a series of choices related to detector usage, target acquisition, dithers and background observations, amongst others. This article discusses the most relevant mode-independent recommendations. Readers are encouraged to visit the JDocs site ${ }^{1}$ for the most up-to-date and dedicated observing mode recommendations.

Further author information: (Send correspondence to Macarena García Marín)

M.M.G.M.: E-mail: Macarena.Garcia.Marin@esa.int, Telephone: 4103385069

Observatory Operations: Strategies, Processes, and Systems VII, edited by Alison B. Peck, Robert L. Seaman, Chris R. Benn, Proc. of SPIE Vol. 10704, 107041I · @ 2018 SPIE CCC code: $0277-786 \mathrm{X} / 18 / \$ 18 \cdot$ doi: $10.1117 / 12.2314319$ 


\section{MIRI DETECTORS USAGE}

The MIRI 1024x1024 pixel $^{2}$ detectors use exclusively the sample-up-the-ramp operations scheme (see ${ }^{4}$ and Fig. 1). In this context, one exposure is a number of sequential integrations within a single command; one integration is a number of non-destructive readout groups as photo-electrons are accumulated, after which the detector undergoes a reset. A group is the product of cycling through all the array pixels. Groups can be obtained in FAST and SLOW mode (2.775 and 23.890 seconds per group, respectively $\left.{ }^{4}\right)$. At the time of setting up a science program observers will have to define all of these variables, making use of the JWST Exposure Time Calculator $\left(\mathrm{ETC}^{11,12}\right)$ to estimate the signal-to-noise (SNR) that will be achieved.

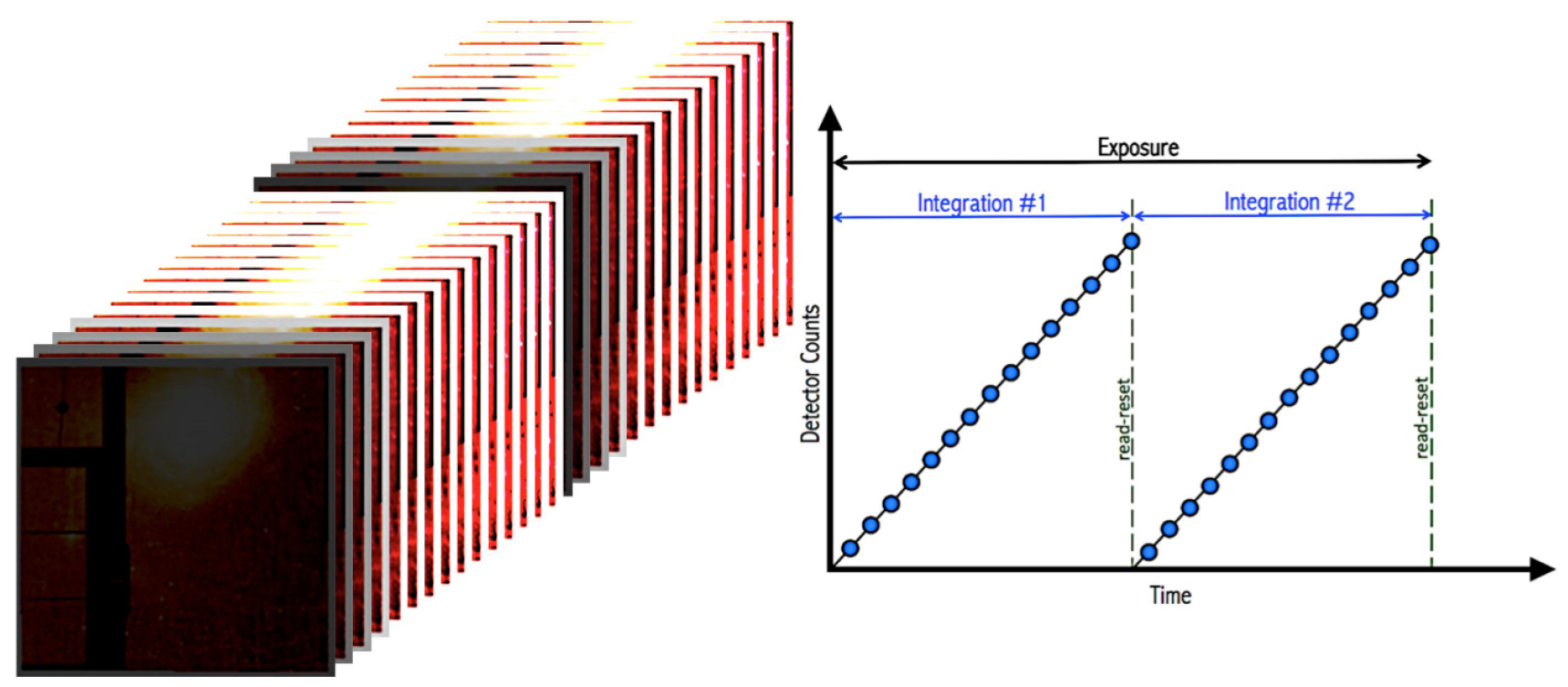

Figure 1. Diagram illustrating the sample-up-the-ramp readout scheme used by the MIRI detectors. Left: Simulated MIRI imager module detector data; each image represents one read/group. The signal builds up until the detector is reset and a new integration starts. In this particular example the exposure consists of 2 integrations, 15 groups per integration. Right: Groups (blue points), integrations and exposures as "seen" by a single illuminated pixel.

To help users navigate this broad parameter space, we have defined a set of detector "Best Practices", common to all MIRI observing modes, that can be summarized in the following set of questions and answers.

- How should I set up the MIRI detectors? Figure 2 gives a general overview of MIRI detectors usage for bright and faint targets.

- Choosing a readout mode: FAST or SLOW? The MIRI detectors use two different readout schemes. ${ }^{4}$ While FAST mode has a finer time sampling that helps to better characterize the detector effects and cosmic-rays, SLOW mode provides about 9 times less data volume. For planning observations FAST is the generally best suited and default mode for imaging, coronagraphy and LRS. SLOW mode, however, will be very useful in low background* imaging parallel observations. For bright sources and high background observations, the MIRI imaging module offers a set of subarrays with finer time sampling (see details in ${ }^{4}$ ).

On the other hand, in MRS observations SLOW mode may deliver slightly lower noise and fewer subtle spatial artifacts, being thus the default mode for this observing mode. Note that SLOW mode integrations should not be shorter that 10 groups $(\sim 240 \mathrm{~s})$, in those cases users should always opt for FAST mode. These default options are not mandatory, and the user is free to change them.

\footnotetext{
* Observers should keep in mind that at longer wavelengths MIRI will be affected by the high thermal background
} coming from the telescope that can potentially saturate the detector in imaging mode. 


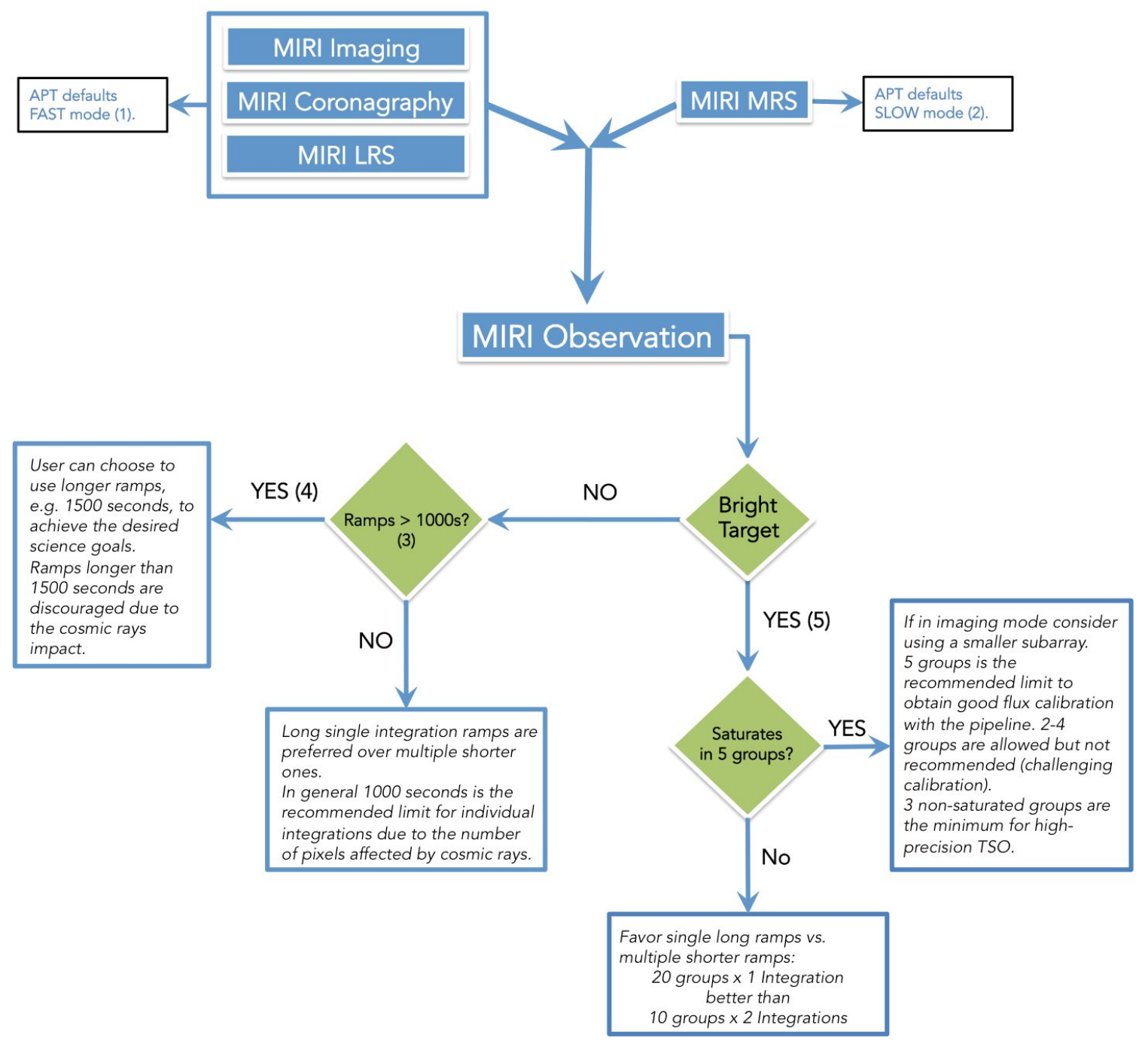

Figure 2. General guidelines on MIRI detectors usage for faint and bright targets. (1) APT is the JWST Astronomer's Proposal Tool. For MIRI imaging SLOW mode is advisable to reduce data volume in low background parallel observations. (2) The user is encouraged to use FAST mode for MRS bright sources observations. SLOW mode integrations should not be shorter than 10 groups (240 s). (3) Users should rely on the SNR and saturation levels as reported by the JWST/ETC. (4) Example use case: high-redshift spectroscopic observations. (5) For bright targets observers should generally rely on FAST mode readout. Having one group saturated at the end of the integration will help recovering information in those pixels (see saturation discussion in Sec. 2. This strategy will also improve the SNR in the non-saturated pixels.

- What is the ideal length of an integration? The optimal length of an integration will depend on the specific science case and required SNR. In general long integrations are preferable. This is because the initial groups are the most affected by non-ideal (but known) transient effects. ${ }^{4}$ Longer integrations are dominated by other more standard departures from ideal behavior, such as non-linearity. 100 groups in FAST ( 278 seconds) and 25 in SLOW mode ( 590 seconds) are the suggested starting point values; users should then work with the JWST ETC and shorten or lengthen the integration to achieve the desired SNR.

- What is the minimum length of an integration? 5 groups is the recommended lower limit to obtain good flux calibration with the Space Telescope Science Institute (STScI) calibration pipeline ${ }^{13}$. 3 nonsaturated groups are the minimum number to achieve high-precision TSO (time series observations ${ }^{14,15}$ ). 
When using fewer than 5 groups, and if photometric accuracy is required, the observers should obtain a calibration star with exactly the same exposure parameters. This is not necessary for TSO, where repeatability precision is the main requirement. Single group integrations are not allowed for MIRI science observations.

- What is the maximum recommended length of an integration? Currently there is no imposed limit on the MIRI integration lengths ${ }^{\dagger}$. Due to the predicted impact of cosmic-rays hits, for most science cases the recommended maximum integration is 1000 seconds. Independent studies by the MIRI team that use both a Monte-Carlo and an analytical approach, and take advantage of cosmic-rays information from the Spitzer data, predict that for integrations longer than 1000 seconds about $60 \%$ of the detector pixels will be affected by cosmic-rays. This not only includes the pixels that are directly impacted by a cosmic-ray, but also the four adjacent pixels (see Fig. 3). The calibration pipeline will be able to recover information from these pixels using both segments of the ramp, which is one of the key advantages of the sample-up-the-ramp operation scheme, but there still may be an impact in the SNR. There are, however, science cases that will need longer integrations to achieve the science goals (e.g. high-redshift spectroscopic observations). In such cases, integrations longer than 1500 seconds are discouraged. A reset is needed after such a long integration to re-impose the nominal detector biases. In many cases the integration length will be limited by the saturation limits of sources within the field (see below) and/or the need to separate the observation into a minimum number of dither positions.
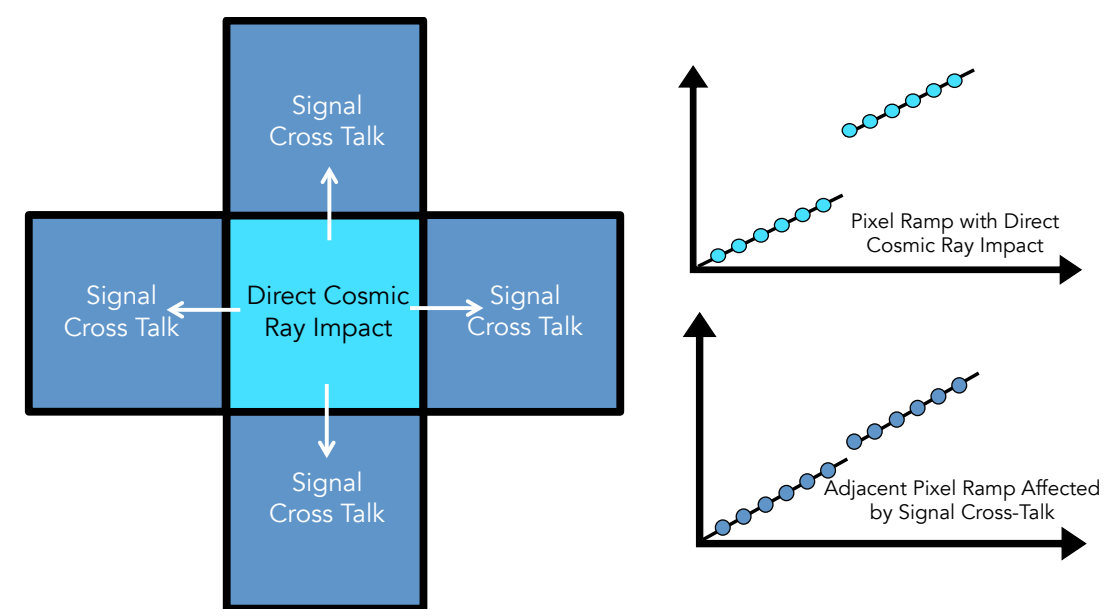

Figure 3. Left: Illustration of a single MIRI pixel hit by a cosmic-ray; the four adjacent pixels are affected by cross-talk due to inter-pixel capacitance. Top Right: Pixel ramp that has undergone a direct impact. Bottom Right: Adjacent pixel ramp affected by cross-talk from the cosmic hit as well.

- Should I use single or multiple integrations? When using multiple integrations, the first integration of a series is always different than the rest, because it is free of transient reset effects affecting the initial groups in subsequent integrations. The time needed to set up the exposure and instrument allows for multiple resets that clear up the features generated by those effects. In comparison, between integration within the same exposure there is only one reset, and thus some non-ideal transient behavior has a higher impact. These effects are greatly reduced by the data processing pipeline, but the greater the number of samples per integration the more accurate the result will be. It is thus best to favor single long integrations over multiple short ones. As with all recommendations users should keep in mind their particular science case: for instance, TSO programs on bright targets will need to use short multiple integrations to achieve their science goals.

\footnotetext{
${ }^{\dagger}$ Other than the 10000 seconds exposure limit to adjust the observatory High Gain Antenna pointing,. ${ }^{16}$ This is valid for all observations except TSO.
} 
- How will saturation impact my data? The MIRI detectors readout scheme allows use of partially saturated data, that is, integrations where only some groups are saturated. In fact, sometimes having some degree of saturation can be beneficial to extract information from the maximum possible dynamic range: in the current calibration pipeline ${ }^{13}$ the last group of each integration will be automatically discarded. Having thus one group saturated at the end of the integration will help recovering information in those pixels. This strategy will also improve the SNR in the unsaturated regions. Saturating more than one group does not provide direct benefits, but it does not invalidate data on the initial part of the integration. Observers should use the JWST/ETC to judge saturation limits. It is worth noting that integrations 20-30\% above the saturation limit as reported by the JWST/ETC should be usable, but by doing this observers are assuming a risk. Definitive saturation limits will be only known on-orbit.

\section{TARGET ACQUISITION CONSIDERATIONS}

Table 1 provides an overview of the MIRI observing modes and their target acquisition (TA) capabilities: TA is mandatory in coronagraphy and LRS slitless observations, where there is requirement of high precision/repeatability in the source location. More TA-related information is available in JDocs. ${ }^{17}$

Table 1. Availability of Target Acquisition for all MIRI modes

\begin{tabular}{|l|l|}
\hline MIRI Mode & Target Acquisition \\
\hline Imaging & Under consideration for Cycle 1 \\
\hline Coronagraphy & Mandatory \\
\hline LRS Slit & Optional \\
\hline LRS Slitless (TSO) & Mandatory \\
\hline MRS & Optional \\
\hline
\end{tabular}

For cases where TA is optional, observers should evaluate the need for TA and base the decision on (1) the JWST expected pointing performance ${ }^{18}$ (see Sec. 3.1) and (2) TA feasibility (see Sec.3.2).

\subsection{JWST Expected Pointing Performance}

The pre-launch estimated absolute fine pointing accuracy (without TA) for the MIRI MRS is 0.45 " ( $1 \sigma$ radial error). The MRS represents the worst case scenario, as it is the instrument aperture most distant from the guide star. This uncertainty is dominated by the guide star catalog position errors and pointing errors due to roll control. Users should consider the impact of this uncertainty combined with the size of the MRS FOV $(3.3$ " $\times$ 3.7", slice width 0.176 " at the shortest wavelength) and LRS slit $(4.7 " \times 0.51$ ") to decide whether TA is necessary.

\subsection{Target Acquisition Feasibility}

Target acquisition is an on-board process that, in a nutshell, consists of the following steps:

1. For MIRI, TA is always done using the imager detector. The raw image is obtained, background-subtracted and flat-fielded on board.

2. The algorithm finds the brightest $3 \times 3$ pixels $^{\ddagger}$ region on the detector region of interest (ROI) where the TA source is located. This ROI is $64 \times 64$ pixels for all cases except for:

- Coronagraphy, where there is a second TA in a smaller $(16 \times 16$ pixels $)$ ROI.

- The smallest imager subarray (SUB64) where the ROI is $32 \times 32$ pixels.

The location of the ROI on the detector changes depending on the observing mode. The checkbox size $(3 \times 3$ pixels $)$ has been defined to encompass the imager PSF at the MIRI TA filters.

\footnotetext{
${ }^{\ddagger}$ MIRI detectors plate scale $0.11 \mathrm{arcsec} / \mathrm{pixel}$
} 
3. The algorithm then performs a location refinement by calculating the centroid in the previously located brightest area.

4. Once the centroid is located, the required offset to place the science target in the science aperture is calculated.

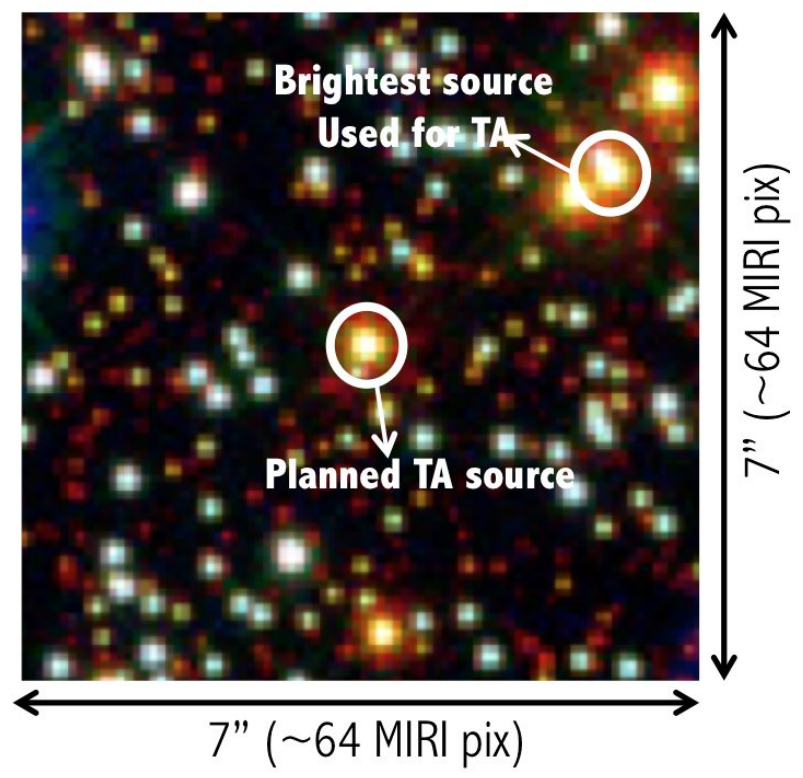

Figure 4. Target acquisition environment illustration. In this example of a rich field, even if the observer selects the central star as a TA target, the centroid algorithm will be performed in the brightest star of the field (top right). Users should base their TA source selection on MIR data to avoid biases due to the use of other SED regimes. Omega Centauri Image Credit: NASA, ESA and the Hubble SM4 ERO team.

The successful completion of the TA procedure will thus depend on the following factors:

- The user-selected TA target must be a point source.

- The TA target has to be the brightest source in the entire ROI, in the filter used for TA (see Fig 4). If that requirement is not met the algorithm will "lock" on to the brightest (by any factor) $3 \times 3$ pixel region within the defined ROI, even if it is part of an extended emission region. This will result in TA being performed on a target not selected by the user, or unsuccessful TA.

If TA is performed on the wrong target, there will be an offset between the required and observed coordinates which is beyond the control of the observer.

Observers are encouraged to carefully select the TA target, and to base that selection on mid-infrared data whenever possible, so that there are no close brighter neighbors.

\section{DITHERING}

As for previous mid-infrared space observatories dithering is highly recommended to achieve the best quality results for all MIRI modes, with notable exceptions like TSO. Dithering, observing at several positions about the target, creates redundancy in the data where more more than 1 pixel samples the same part of the sky. This can help with PSF sampling, calibration of artifacts such as after bright cosmic-ray strikes, mitigating detector hot or dead pixels and improving the overall calibration. For MIRI imaging a minimum of 4 dither positions is 
recommended where the number of dithers for an observation is also defined by the need to be well sampled at each position (see Fig.2).

JWST users will have a set of predefined dithers to choose from; advice on selecting a specific dither pattern for imaging, ${ }^{19} \mathrm{MRS}^{20}$ and $\mathrm{LRS}^{21}$ observations is available in the quoted JDocs pages.

\section{BACKGROUND OBSERVATIONS}

From 5 to about 12 microns the background seen by MIRI will be dominated by the zodiacal light. From 12 to 28.5 microns the thermal emission from the telescope is the most relevant background component, and steeply increases with wavelength. Detailed information about the JWST background model is available in JDocs. ${ }^{22} \mathrm{~A}$ description of the JWST stray light model has been given by, ${ }^{23}$ zodiacal light measurements based on Spitzer data are provided in. ${ }^{24}$

The need for taking a dedicated background observation will always depend on the particular science case and the impact of the background on the science data, that can be evaluated using the JWST ETC. ${ }^{25}$ The general guideline offered by the MIRI team depends on the nature of the source. Point sources will have the background information included in the science pointing already (with the added redundancy provided by dithered data). In the case of targets that extend over the entire instrument FOV, it is advisable to obtain a separate background pointing in every science spectral configuration and observing period of the scientific program. Observing programs requiring high accuracy (better than 1\%) relative calibrations of extended targets may consider taking background data before and after the science exposures in order to help calibrate any drifts in the thermal background of the telescope.

\section{ACKNOWLEDGMENTS}

MIRI draws on the scientific and technical expertise of the following organisations: Ames Research Center, USA; Airbus Defence and Space, UK; CEA-Irfu, Saclay, France; Centre Spatial de Liége, Belgium; Consejo Superior de Investigaciones Científicas, Spain; Carl Zeiss Optronics, Germany; Chalmers University of Technology, Sweden; Danish Space Research Institute, Denmark; Dublin Institute for Advanced Studies, Ireland; European Space Agency, Netherlands; ETCA, Belgium; ETH Zurich, Switzerland; Goddard Space Flight Center, USA; Institute d'Astrophysique Spatiale, France; Instituto Nacional de Técnica Aeroespacial, Spain; Institute for Astronomy, Edinburgh, UK; Jet Propulsion Laboratory, USA; Laboratoire d'Astrophysique de Marseille (LAM), France; Leiden University, Netherlands; Lockheed Advanced Technology Center (USA); NOVA Opt-IR group at Dwingeloo, Netherlands; Northrop Grumman, USA; Max-Planck Institut für Astronomie (MPIA), Heidelberg, Germany; Laboratoire d'Etudes Spatiales et d'Instrumentation en Astrophysique (LESIA), France; Paul Scherrer Institut, Switzerland; Raytheon Vision Systems, USA; RUAG Aerospace, Switzerland; Rutherford Appleton Laboratory (RAL Space), UK; Space Telescope Science Institute, USA; Toegepast-Natuurwetenschappelijk Onderzoek (TNO-TPD), Netherlands; UK Astronomy Technology Centre, UK; University College London, UK; University of Amsterdam, Netherlands; University of Arizona, USA; University of Bern, Switzerland; University of Cardiff, UK; University of Cologne, Germany; University of Ghent; University of Groningen, Netherlands; University of Leicester, UK; University of Leuven, Belgium; University of Stockholm, Sweden; Utah State University, USA. A portion of this work was carried out at the Jet Propulsion Laboratory, California Institute of Technology, under a contract with the National Aeronautics and Space Administration.

\section{REFERENCES}

[1] STScI, MIRI Recommended Strategies (2016 (updated November 30, 2017)). https://jwst-docs.stsci. edu/display/JPP/MIRI+Recommended+Strategies/.

[2] Wright, G. S., Wright, D., Goodson, G. B., Rieke, G. H., Aitink-Kroes, G., Amiaux, J., Aricha-Yanguas, A., Azzollini, R., Banks, K., Barrado-Navascues, D., Belenguer-Davila, T., Bloemmart, J. A. D. L., Bouchet, P., Brandl, B. R., Colina, L., Detre, Ö., Diaz-Catala, E., Eccleston, P., Friedman, S. D., García-Marín, M., Güdel, M., Glasse, A., Glauser, A. M., Greene, T. P., Groezinger, U., Grundy, T., Hastings, P., Henning, T., Hofferbert, R., Hunter, F., Jessen, N. C., Justtanont, K., Karnik, A. R., Khorrami, M. A., Krause, O., Labiano, A., Lagage, P.-O., Langer, U., Lemke, D., Lim, T., Lorenzo-Alvarez, J., Mazy, E., McGowan, N., 
Meixner, M. E., Morris, N., Morrison, J. E., Müller, F., rgaard-Nielson, H.-U. N., Olofsson, G., O’Sullivan, B., Pel, J.-W., Penanen, K., Petach, M. B., Pye, J. P., Ray, T. P., Renotte, E., Renouf, I., Ressler, M. E., Samara-Ratna, P., Scheithauer, S., Schneider, A., Shaughnessy, B., Stevenson, T., Sukhatme, K., Swinyard, B., Sykes, J., Thatcher, J., Tikkanen, T., van Dishoeck, E. F., Waelkens, C., Walker, H., Wells, M., and Zhender, A., "The mid-infrared instrument for the james webb space telescope, ii: Design and build," PASP 127, 595 (2015).

[3] Rieke, G. H., Ressler, M. E., Morrison, J. E., Bergeron, L., Bouchet, P., García-Marín, M., Greene, T. P., Regan, M. W., Sukhatme, K. G., and Walker, H., "The mid-infrared instrument for the james webb space telescope, vii: The miri detectors," PASP 127, 665 (2015).

[4] Ressler, M. E., Sukhatme, K. G., Franklin, B. R., Mahoney, J. C., Thelen, M. P., Bouchet, P., Colbert, J. W., Cracraft, M., Dicken, D., Gastaud, R., Goodson, G. B., Eccleston, P., Moreau, V., Rieke, G. H., and Schneider, A., "The mid-infrared instrument for the james webb space telescope, viii: The miri focal plane system," PASP 127, 675 (2015).

[5] Bouchet, P., García-Marín, M., Lagage, P.-O., Amiaux, J., Auguéres, J.-L., Bauwens, E., Blommaert, J. A. D. L., Chen, C. H., Detre, Ö. H., Dicken, D., Dubreuil, D., Galdemard, P., Gastaud, R., Glasse, A., Gordon, K. D., Gougnaud, F., Guillard, P., Justtanont, K., Krause, O., Leboeuf, D., Longval, Y., Martin, L., Mazy, E., Moreau, V., Olofsson, G., Ray, T. P., Rees, J.-M., Renotte, E., Ressler, M. E., Ronayette, S., Salasca, S., Scheithauer, S., Sykes, J., Thelen, M. P., Wells, M., Wright, D., and Wright, G. S., "The mid-infrared instrument for the james webb space telescope, iii: Mirim, the miri imager," PASP 127, 612 (2015).

[6] Boccaletti, A., Lagage, P.-O., Baudoz, P., Beichman, C., Bouchet, P., Cavarroc, C., Dubreuil, D., Glasse, A., Glauser, A. M., Hines, D. C., Lajoie, C.-P., Lebreton, J., Perrin, M. D., Pueyo, L., Reess, J. M., Rieke, G. H., Ronayette, S., Rouan, D., Soummer, R., and Wright, G. S., "The mid-infrared instrument for the james webb space telescope, v: Predicted performance of the miri coronagraphs," PASP 127, 633 (2015).

[7] Kendrew, S., Scheithauer, S., Bouchet, P., Amiaux, J., Azzollini, R., Bouwman, J., Chen, C. H., Dubreuil, D., Fischer, S., Glasse, A., Greene, T. P., Lagage, P.-O., Lahuis, F., Ronayette, S., Wright, D., and Wright, G. S., "The mid-infrared instrument for the james webb space telescope, iv: The low-resolution spectrometer," PASP 127, 623 (2015).

[8] Wells, M., Pel, J.-W., Glasse, A., Wright, G. S., Aitink-Kroes, G., Azzollini, R., Beard, S., Brandl, B. R., Gallie, A., Geers, V. C., Glauser, A. M., Hastings, P., Henning, T., Jager, R., Justtanont, K., Kruizinga, B., Lahuis, F., Lee, D., Martinez-Delgado, I., Martínez-Galarza, J. R., Meijers, M., Morrison, J. E., Müller, F., Nakos, T., O’Sullivan, B., Oudenhuysen, A., Parr-Burman, P., Pauwels, E., Rohloff, R.-R., Schmalzl, E., Sykes, J., Thelen, M. P., van Dishoeck, E. F., Vandenbussche, B., Venema, L. B., Visser, H., Waters, L. B. F. M., and Wright, D., "The mid-infrared instrument for the james webb space telescope, vi: The medium resolution spectrometer," PASP 127, 646 (2015).

[9] Glasse, A., Rieke, G. H., Bauwens, E., García-Marín, M., Ressler, M. E., Rost, S., Tikkanen, T. V., Vandenbussche, B., and Wright, G. S., "The mid-infrared instrument for the james webb space telescope, ix: Predicted sensitivity," PASP 127, 686 (2015).

[10] Rieke, G. H., Wright, G. S., Böker, T., Bouwman, J., Colina, L., Glasse, A., Gordon, K. D., Greene, T. P., Güdel, M., Henning, T., Justtanont, K., Lagage, P.-O., Meixner, M. E., Nørgaard-Nielsen, H.-U., Ray, T. P., Ressler, M. E., van Dishoeck, E. F., and Waelkens, C., "The mid-infrared instrument for the james webb space telescope, i: Introduction," PASP 127, 584 (2015).

[11] Pontoppidan, K. M. and Pickering, T. E. and Laidler, V. G. and Gilbert, K. and Sontag, C. D. and Slocum, C. and Sienkiewicz, M. J. and Hanley, C. and Earl, N. M. and Pueyo, L. and Ravindranath, S. and Karakla, D. M. and Robberto, M. and Noriega-Crespo, A. and Barker, E. A., P. A., "Pandeia: a multimission exposure time calculator for jwst and wfirst," in [Observatory Operations: Strategies, Processes, and Systems VI], Benn, A. B. P. R. L. S. C. R., ed., Proc. SPIE 9910, 991016 (2016).

[12] STScI, JWST Exposure Time Calculator Overview (2016 (updated December 30, 2016)). https:// jwst-docs.stsci.edu/display/JPP/MIRI+Recommended+Strategies/.

[13] Gordon, K. D., Chen, C. H., Anderson, R. E., Azzollini, R., Bergeron, L., Bouchet, P., Bouwman, J., Cracraft, M., Fischer, S., Friedman, S. D., García-Marín, M., Glasse, A., Glauser, A. M., Goodson, G. B., Greene, T. P., Hines, D. C., Khorrami, M. A., Lahuis, F., Lajoie, C.-P., Meixner, M. E., Morrison, J. E., 
O’Sullivan, B., Pontoppidan, K. M., Regan, M. W., Ressler, M. E., Rieke, G. H., Scheithauer, S., Walker, H., and Wright, G. S., "The mid-infrared instrument for the james webb space telescope, x: Operations and data reduction," PASP 127, 696 (2015).

[14] STScI, MIRI-Specific Time-Series Observations (2016 (updated Mach 7, 2018)). https://jwst-docs. stsci.edu/display/JPP/MIRI-Specific+Time-Series+Observations.

[15] Kendrew, S., Dicken, D., Bouwman, J., Garcia-Marin, M., Greene, T., Lagage, P.-O., Ressler, M., Batalha, N. M., Bean, J. L., Crouzet, N., Kreidberg, L., Stevenson, K. B., Glasse, A., Wright, G., and Rieke, G. H., Time Series Observations with the Mid-IR Instrument (MIRI) on the James Webb Space Telescope (2018). Proc. SPIE, 10698, 133.

[16] STScI, JWST Communications Subsystem (2016 (updated May 17, 2017)). https://jwst-docs.stsci. edu/display/JTI/JWST+Communications+Subsystem.

[17] STScI, MIRI Target Acquisitions (2016 (updated January 2, 2017)). https://jwst-docs.stsci.edu/ display/JTI/MIRI+Target+Acquisitions.

[18] STScI, JWST Pointing Performance (2016 (updated May 31, 2017)). https://jwst-docs.stsci.edu/ display/JTI/JWST+Pointing+Performance.

[19] STScI, MIRI Imaging Recommended Strategies (2016 (updated Nov 30, 2017)). https://jwst-docs.stsci. edu/display/JPP/MIRI+Imaging+Recommended+Strategies.

[20] STScI, MIRI MRS Recommended Strategies (2016 (updated Nov 30, 2017)). https://jwst-docs.stsci. edu/display/JPP/MIRI+MRS+Recommended+Strategies.

[21] STScI, MIRI LRS Recommended Strategies (2016 (updated Nov 30, 2017)). https://jwst-docs.stsci. edu/display/JPP/MIRI+LRS+Recommended+Strategies.

[22] STScI, JWST Background Model (2016 (updated Nov 15, 2017)). https://jwst-docs.stsci.edu/ display/JPP/JWST+Background+Model.

[23] Lightsey, P. A., "Stray light field dependence for the james webb space telescope," in [Space Telescopes and Instrumentation 2016: Optical, Infrared, and Millimeter Wave], J.W. A. den Herder, T. Takahashi, M. B., ed., Proc. SPIE 9904, 99040A (2016).

[24] Krick, J. E., Glaccum, W. J., Carey, S. J., Lowrance, P. J., Surace, J. A., Ingalls, J. G., Hora, J. L., and Reach, W. T., "A spitzer/irac measure of the zodiacal light," ApJ 754, 53 (2012).

[25] STScI, JWST ETC Backgrounds (2016 (updated Nov 29, 2017)). https://jwst-docs.stsci.edu/ display/JPP/JWST+ETC+Backgrounds. 